\title{
Bestrahlung aller Lymphknoten lohnt sich
}

Eine akzelerierte integrierte InvolvedField-Radiotherapie (IFRT) gefolgt von einer Bestrahlung des gesamten lymphatischen Systems (TLI) und einer hochdosierten Chemotherapie ist eine wirksame, praktikable und sichere Salvage-Strategie für Patienten mit einem rezidivierten oder refraktären Hodgkin-Lymphom (HL).

Zwischen November 1985 und Juli 2008 unterzogen sich 186 zuvor nicht bestrahlte Patienten mit refraktärem oder rezidiviertem HL dieser Behandlung. Nach einer normaldosierten SalvageChemotherapie wurde eine akzelerierte IFRT (18-20 Gy) auf die rezidivierten oder refraktären Tumororte gegeben, gefolgt von TLI (15-18 Gy) und hochdosierter Chemotherapie. Nach einem mittleren Follow-up der Überlebenden von 57 Monaten betrugen die 5- bzw. 10-Jahres-Gesamtüberlebensraten 68 bzw. 56\%. Die ereignisfreien 5- bzw. 10-Jahres-Überlebensraten lagen bei 62 bzw. $56 \%$; und die kumulativen Inzidenzen von HL-zugehörigen Todesfällen lagen nach fünf bzw. zehn Jahren bei 21 bzw. $29 \%$.

Einer Multivarianzanalyse zufolge war das komplette Ansprechen auf die Salvage-Chemotherapie mit einem verbesserten Gesamtüberleben und ereignisfreien Überleben assoziiert. Eine primäre refraktäre Krankheit und eine extranodale Erkrankung waren dagegen mit einem ungünstigen krankheitsspezifischen Überleben assoziiert.

Bei acht Patienten entwickelte sich eine kardiale Toxizität vom Schweregrad 3

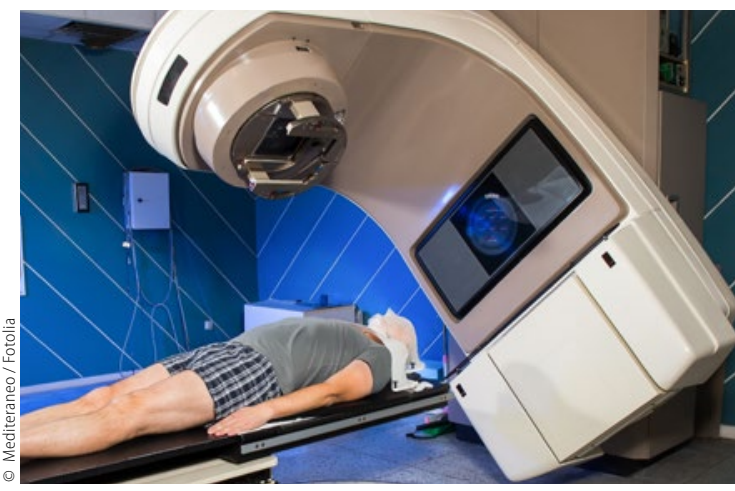

Ohne Bestrahlung käme es beim HodgkinLymphom häufig zu Rezidiven.

oder mehr, die mit drei Todesfällen einherging. Sekundäre Malignitäten entstanden bei zehn Patienten, von denen fünf starben.

Christina Berndt

Rimner A et al. Accelerated Total Lymphoid Irradiation-containing Salvage Regimen for Patients With Refractory and Relapsed Hodgkin Lymphoma: 20 Years of Experience. Int J Radiat Oncol Biol Phys. 2017;97(5):1066-76.

\section{Adjuvantes Temozolomid auch bei schlechter Prognose sinnvoll}

Eine adjuvante Temozolomid-Therapie ist bei anaplastischen Gliomen ohne 1p/19qKodeletion hilfreich: Die progressionsfreie Überlebenszeit lässt sich damit verdoppeln. Dies ergab die Studie CATNON mit 745 Patienten mit frisch diagnostiziertem anaplastischem Gliom ohne Kodeletion. Alle Patienten wurde primär operiert. Anschließend wurde in vier Gruppen randomisiert: In Gruppe 1 wurde nur bestrahlt (59,4 Gy in 33 Fraktionen), Gruppe 2 erhielt gleichzeitig mit der Radiatio eine Temozolomid-Chemotherapie (täglich $75 \mathrm{mg} / \mathrm{m}^{2}$ für maximal 7 Wochen), Gruppe 3 erhielt eine adjuvante TemozolomidTherapie (12 Zyklen mit 150-200 mg/m² Temozolomid, Tag 1-5, q4w), beginnend vier Wochen nach dem Ende der Bestrahlung. Gruppe 4 wurde zeitgleich zur Radiatio sowie adjuvant mit Temozolomid behandelt.

Bis zum Stichtag im Mai 2015 waren die Patienten im Median 27 Monate nachuntersucht worden. Bei $46 \%$ der Patienten war es zu einer Tumorprogression gekommen. Bei $39 \%$ mit und $54 \%$ ohne adjuvante Temozolomid-Behandlung. Im Median lebten Patienten mit ad- juvanter Therapie 43 Monate progressionsfrei, ohne waren es nur 19 Monate.

Mit adjuvanter Temozolomid-Behandlung lag die Sterberate bei $25 \%$, ohne adjuvante Therapie bei $35 \%$. Relativ betrachtet war die Sterberate unter Berücksichtigung einer Reihe von Begleitfaktoren und Tumorcharakteristika damit unter adjuvantem Temozolomid um 35\% reduziert. Nach fünf Jahren waren $43 \%$ mit und nur $24 \%$ ohne adjuvante Therapie noch ohne Zeichen einer Tumorprogression am Leben.

Thomas Müller

van den Bent MJ et al. Interim results from the CATNON trial (EORTC study 26053-22054) of treatment with concurrent and adjuvant temozolomide for 1p/19q non-co-deleted anaplastic glioma: a phase 3, randomised, open-label intergroup study. Lancet. 2017:390(10103):1645-53.

\section{kurz notiert}

\section{Antikoagulans reduziert die Krebsinzidenz}

Bei über 50-Jährigen scheint die Anwendung des Vitamin-K-Antagonisten Warfarin mit einem geringeren Krebsrisiko assoziiert zu sein. Daten einer aktuellen Studie zufolge gingen das Gesamtkrebsrisiko (Inzidenzratenverhältnis [IRR] 0,84) wie auch das Risiko für die drei häufigsten Tumoren (Prostatakrebs: IRR 0,69; Lungenkrebs: IRR 0,80; Brustkrebs bei Frauen: IRR 0,90) bei Patienten mit Warfarin-Therapie im Vergleich zu Personen, die nicht mit Warfarin behandelt wurden, zurück [Haaland GS et al. JAMA Intern Med. 2017; Nov 6 (Epub ahead of print)]. Bei Patienten, die Warfarin zur Behandlung von Vorhofflimmern oder Vorhofflattern erhielten, sanken das Gesamtkrebsrisiko (IRR 0,62) sowie das Risiko von Lungen-, Prostata-, Brust- und Darmkrebs. In der Beobachtungsstudie waren Daten von 1,25 Millionen Menschen in Norwegen analysiert worden, die zwischen 1924 und 1954 geboren waren und in das nationale norwegische Register aufgenommen wurden. Im Tiermodell inhibierte Warfarin die AXL-Rezeptor-Tyrosinkinaseabhängige Tumorentstehung und verstärkte die Antitumorimmunantwort in Konzentrationen, die unterhalb der Dosen lagen, die für die Antikoagulation verwendet werden. Judith Neumaier 